\title{
Medical Image of the Week: Alpha Intrusion into REM Sleep
}

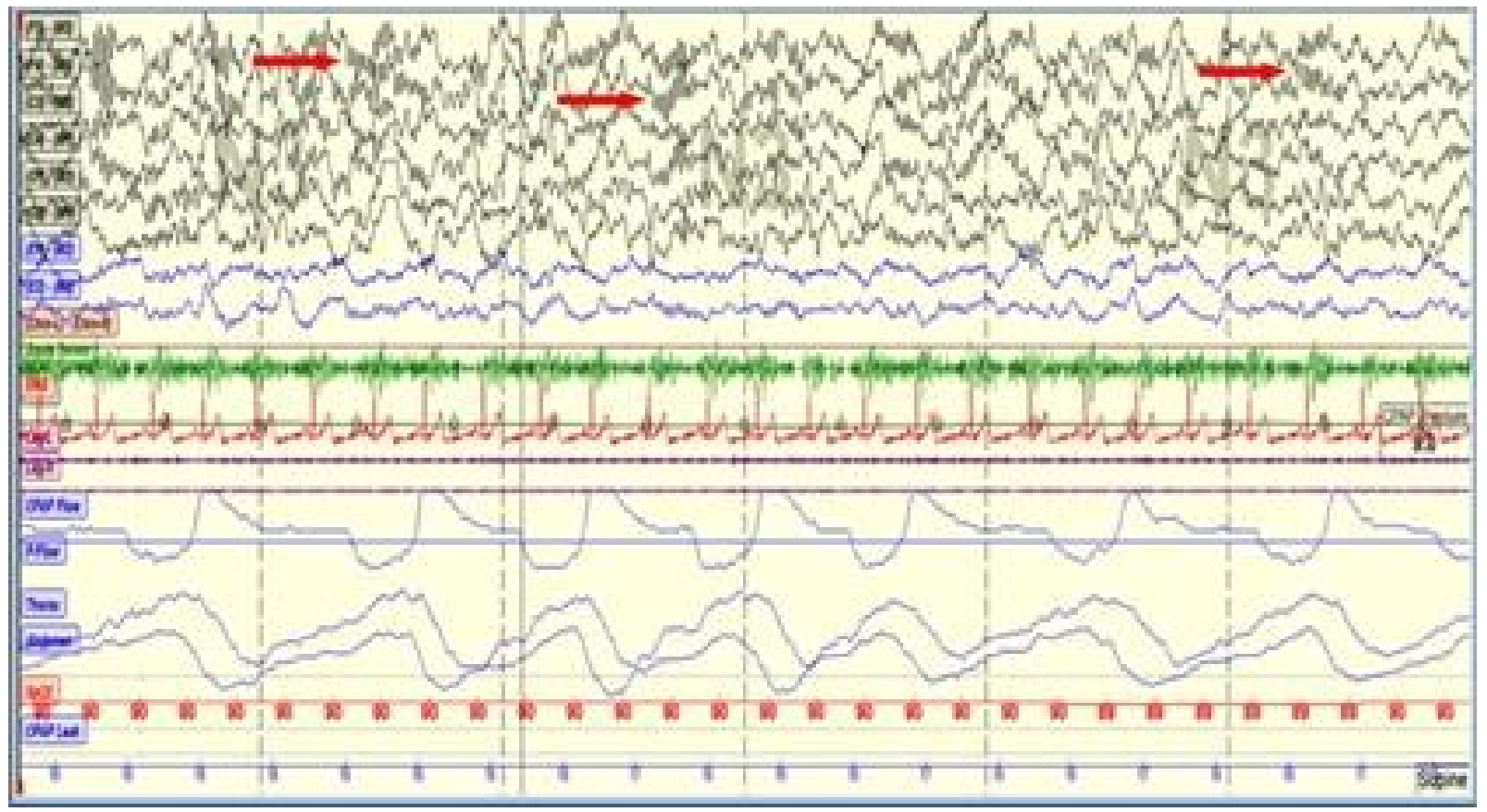

Figure 1. 30-second epoch during NREM sleep (Stage N3). The red arrows show alpha waves (8-12 Hz frequency) on delta waves $(0.5-2 \mathrm{~Hz})$, most prominently seen in the frontal and central EEG leads.

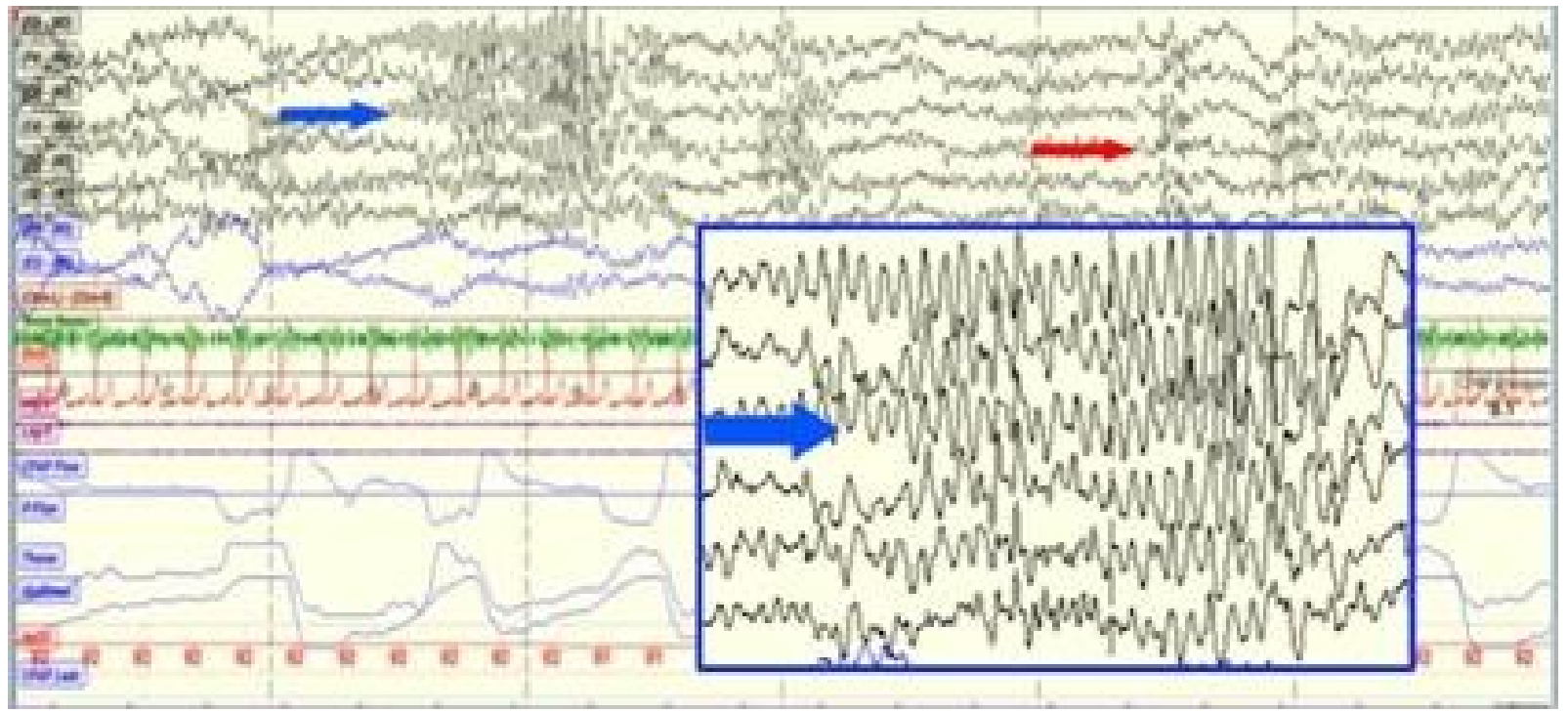

Figure 2. 30 second epoch during REM sleep showing bursts of alpha activity during REM sleep (red arrow) with hypersynchronous theta wave activity (4-7 Hz) (blue arrow). 
A 45-year-old woman with a past medical history of hypertension and chronic headaches was referred to the sleep laboratory for high clinical suspicion for sleep apnea based on a history of snoring, witnessed apnea and excessive daytime sleepiness. An overnight sleep study was performed. Images during N3 Sleep and REM sleep are shown (Figures 1 and 2).

Alpha intrusion in delta sleep is seen in patients with fibromyalgia, depression, chronic fatigue syndrome, anxiety disorder, and primary sleep disorders like psychophysiological insomnia, obstructive sleep apnea, circadian disorders and narcolepsy (1).

Bursts of alpha waves during REM sleep may be more common during phasic REM than tonic REM. The REM alpha bursts (alpha activity lasting at least 3 seconds without an increase in EMG amplitude) may represent microarousals (2).

Hypersynchronous theta activity should be differentiated from the spike and waveform activity seen in seizures.

Safal Shetty MD, Tam Le, MD Banner University Medical Center

Tucson, AZ

\section{References}

1. Jaimchariyatam N, Rodriguez CL, Budur K. Prevalence and correlates of alpha-delta sleep in major depressive disorders. Innov Clin Neurosci. 2011;8(7):35-49. [PubMed]

2. Cantero JL, Atienza M. Alpha burst activity during human REM sleep: descriptive study and functional hypotheses. Clin Neurophysiol. 2000;111(5):909-15. [CrossRef] [PubMed] 